\title{
An approach to acute pulmonary embolism in the primary emergency care environment
}

\author{
M Naidoo \\ Discipline of Family Medicine, School of Nursing and Public Health, University of KwaZulu-Natal
}

Pulmonary embolism (PE) is a common emergency presenting among high risk patients. It is important to have a structured approach to clinical assessment, diagnosis and management of this potentially life-threatening emergency. Clinical and management pathways have improved over the last 10 years as clear guidelines have emerged. Newer diagnostic modalities have become available and will hopefully improve the pick-up rate of PE and reduce the morbidity and mortality. High level evidence presented by the American Society of Haematology and the European Society of Cardiology forms the basis for this review.

\section{Background}

Pulmonary embolism (PE) and deep vein thrombosis (DVT) are clinical manifestations of venous thrombo-embolic disease (VTE) and both have similar risk factors. Pulmonary embolism is thought to be common and has been estimated to have a prevalence of $0.4 \%$ among hospitalised patients in the United States of America (US). The clinical presentation is not always classic as $50 \%$ of patients with PE were found to be asymptomatic when patients with proximal DVT were investigated. A communitybased study in France estimated the annual incidence of PE as $6 / 10000$ population per year, however these figures may underestimate the true incidence as post mortem data was not used to validate the figures. ${ }^{1}$ Approximately $79 \%$ of patients with PE have associated DVT in the limbs so it is important to exclude PE by investigating appropriately. ${ }^{2}$ Treated patients with no right ventricular dysfunction have a mortality rate of $2 \%$ which increases to $30 \%$ in patients who present with shock. Patients who present with cardiac arrest have a mortality rate of $65 \%{ }^{3}$ It is important for a primary care physician to identify high risk patients, consider the diagnosis of PE in acutely ill patients and follow a structured approach to diagnosis and management.

The Padau predication and Wells scoring charts help quantify the patients risk for VTE by using recognised risk factors which include the presence of active carcinoma, immobility, history of VTE, thrombophilia, recent trauma or surgery, advanced age, heart or respiratory failure, stroke or myocardial infarction, obesity, hormonal treatment and active infection. ${ }^{4}$

The manifestations of PE are predominately haemodynamic and become apparent when greater than $30-50 \%$ of the pulmonary arterial bed is obstructed by emboli. This acutely increases pulmonary artery pressures and causes an overloading effect of the right ventricle (RV) which is not able to cater for the afterload needed for the systemic circulation resulting in electromechanical dissociation and sudden death. Alternatively, acute right heart failure may lead to hypotension, syncope and sudden death. Patients who survive have sympathetic nervous system activation resulting in an inotropic and chronotropic response which aims at stabilising the systemic circulation. ${ }^{1}$

\section{Clinical Presentation}

Having a strong index of suspicion among individuals regarded as high risk is imperative. Having a Padau predication score of $\geq 4$ or a Wells score of $\geq 3$ should make the clinician aware that they may be dealing with PE. ${ }^{4}$ The typical clinical manifestations of patients presenting with PE include leg pain or warmth, dyspnoea, chest pain (often pleuritic in nature), haemoptysis, cough and syncope. Examination finding may include tachycardia, tachypnoea, hypotension, pyrexia, crepitations and wheeze in the lungs and signs of pulmonary hypertension which may include a parasternal heave, a loud $\mathrm{P} 2$, a right sided gallop and distended neck veins. ${ }^{1,2}$ Any patient presenting with shock or hypotension is immediately classified as high risk. ${ }^{5}$ However, none of these symptoms are sensitive and specific enough to rule in/out a diagnosis of PE as outlined by the European Society of Cardiology (ESC) guidelines depicted in Table 1.

To determine the probability of $\mathrm{PE}$, the clinician should use the probability scores based on either the revised Geneva score or the Wells scores which are depicted in Table 2.

A two-level probability is most useful in the outpatient settings.

\section{Investigations}

Electrocardiographic (ECG) signs such as sinus tachycardia, RV strain pattern, a QR pattern in lead V1, the classic S1Q3T3 type and right bundle-branch block may be present and may assist with the diagnosis. ${ }^{1}$ The American Society of Haematology (ASH) recommends using a D-dimer test in patients rated as low or intermediate probability. An age adjusted D-dimer test using cut off values of $500 \mathrm{ug} / \mathrm{L}$ will assist in deciding whether further investigations are needed in a resource constrained environments. ${ }^{7}$ A low or negative D-dimer excludes PE in the low and intermediate categories. The ASH guideline evaluated 53 studies and provided a summary of evidence of the various 
Table 1: Prevalence of symptoms and signs in patients with suspected PE according to final diagnosis 1,5

\begin{tabular}{|c|c|c|}
\hline Clinical features & PE confirmed $(n=1880)$ & PE excluded $(n=528)$ \\
\hline Dyspnoea & $50 \%$ & $51 \%$ \\
\hline Chest pain (pleuritic) & $39 \%$ & $28 \%$ \\
\hline Chest pain (substernal) & $15 \%$ & $17 \%$ \\
\hline Cough & $20 \%$ & $25 \%$ \\
\hline Haemoptysis & $8 \%$ & $4 \%$ \\
\hline Syncope & $6 \%$ & $6 \%$ \\
\hline Signs of DVT & $15 \%$ & $10 \%$ \\
\hline Fever $(>38.50 \mathrm{C})$ & $10 \%$ & $10 \%$ \\
\hline Clinical features & $(n=219)$ & $(n=546)$ \\
\hline Tachypnoea (> 20/min) & $70 \%$ & $68 \%$ \\
\hline Tachycardia (> 100/min) & $26 \%$ & $23 \%$ \\
\hline Cyanosis & $11 \%$ & $9 \%$ \\
\hline
\end{tabular}

Table 2: Wells and revised Geneva scores ${ }^{6,7}$

\begin{tabular}{|c|c|c|c|}
\hline \multicolumn{2}{|c|}{ Wells score } & \multicolumn{2}{|l|}{ Revised Geneva score } \\
\hline Variable & Points & Variable & Points \\
\hline Previous DVT or PE & 1.5 & Age $>65$ years & 1 \\
\hline Recent surgery or immobilization & 1.5 & Previous DVT or PE & 3 \\
\hline Cancer & 1 & Surgery or fracture within 1-month & 2 \\
\hline Haemoptysis & 1 & Active malignancy & 2 \\
\hline Heart rate $>100$ beats/min & 1.5 & Unilateral lower limb pain & 3 \\
\hline Clinical signs of DVT & 3 & Haemoptysis & 2 \\
\hline \multirow[t]{3}{*}{ Alternative diagnosis less likely than PE } & 3 & Heart rate $75-94$ beats/min & 3 \\
\hline & & Heart rate $\geq 95$ beats/min & 5 \\
\hline & & $\begin{array}{l}\text { Pain on lower limb deep vein at palpation and } \\
\text { unilateral oedema }\end{array}$ & 4 \\
\hline Clinical probability (3 levels) & Total & Clinical probability (3 levels) & Total \\
\hline Low $(<5 \%)$ & $0-1$ & & $0-3$ \\
\hline Intermediate ( 20\%) & $2-6$ & & $4-10$ \\
\hline High (> 50\%) & $\geq 7$ & & $\geq 11$ \\
\hline Clinical probability (2 levels) & & Clinical probability ( 2 levels) & \\
\hline PE unlikely & $0-4$ & PE unlikely & $0-3$ \\
\hline PE likely & $>4$ & PE likely & $>3$ \\
\hline
\end{tabular}

diagnostic tests available. These are tabulated in Table 3. The computed tomography pulmonary angiography (CTPA) has long been recognised as the gold standard for diagnosis but is often not readily available in low-resource settings and one may opt not to use the CTPA as the next most appropriate investigation in patients with low or intermediate pre-test probability of $\mathrm{PE}$ (PTP). The challenges of access, cost and radiation exposure may require one to use a more prudent approach. ${ }^{7}$

The ASH recommends a clinical algorithm based on the PTP. Illustrated in Figure 1 is a diagnostic approach to a patient who presents with a high PTP based on the Wells or revised Geneva scores and will allow the primary care physician to make a clinical decision rule (CDR).

Of note in resource constrained environments when CTPA and VQ scan are not readily available is the possibility of diagnosing $\mathrm{PE}$ with a positive D-dimer and a proximal leg ultrasound, which is a relatively easy skill to master in the primary emergency care environment. The expanded use of ultrasound (echocardiography) in the assessment of PE is available in some emergency care settings and one would encourage primary emergency care physicians to acquire such skills. ${ }^{8}$ The Critical Care Society of South Africa offers the short course called the Cardio-Respiratory Ultrasound Experience (CRUSE), which very efficiently teaches emergency care practitioners skills in cardiac ultrasound. The focussed cardiac sonar using an apical four chamber view can quickly asses RV and LV diameters to determine if the normal ratio of 0.6 is exceeded. ${ }^{9}$ The negative predicative value of bedside echocardiography in a patient with shock or hypotension where there are no echocardiographic signs of RV overload or dysfunction will allow a primary care physician to exclude PE as a differential diagnosis. ${ }^{1}$ Adjunctive investigations such as chest radiography and arterial blood gas analysis have poor specificity but may be important in evaluating severity of the PE. ${ }^{1}$ 
Table 3. Summary of results of studies informing sensitivity and specificity of tests for diagnosis of $\mathrm{PE}^{7}$

\begin{tabular}{lccc}
\hline \multicolumn{1}{c}{ Test } & $\begin{array}{c}\text { No. of participants } \\
\text { (studies) }\end{array}$ & Sensitivity (95\% CI) & Specificity (95\% CI) \\
\hline CTPA & $3929(15)$ & $0.93(0.88-0.96)$ & $0.98(0.96-0.99)$ \\
\hline D-dimer & $20568(30)$ & $0.97(0.96-0.98)$ & $0.39(0.36-0.43)$ \\
Age-adjusted D-dimer & $2885(1)$ & $0.99(0.98-1.00)$ & $0.47(0.45-0.49)$ \\
\hline Proximal ultrasound & $1715(7)$ & $0.49(0.31-0.66)$ & $0.96(0.95-0.98)$ \\
VQ 1 & $3994(13)$ & $0.58(0.50-0.66)$ & $0.98(0.96-0.99)$ \\
VQ 2 & $3994(13)$ & $0.98(0.95-0.99)$ & $0.36(0.27-0.45)$ \\
VQ 3 & $3994(13)$ & $0.96(0.91-0.98)$ & $0.95(0.89-0.98)$
\end{tabular}

$\mathrm{Cl}=$ confidence interval; $\mathrm{VQ}=$ Ventilation perfusion scan; $\mathrm{VQ}$ 1, high probability scan interpreted as positive, low/nondiagnostic/normal scan interpreted as negative; VQ 2, high/low/nondiagnostic probability scan interpreted as positive, normal scan interpreted as negative; VQ 3, high probability scan interpreted as positive, normal scan interpreted as negative.

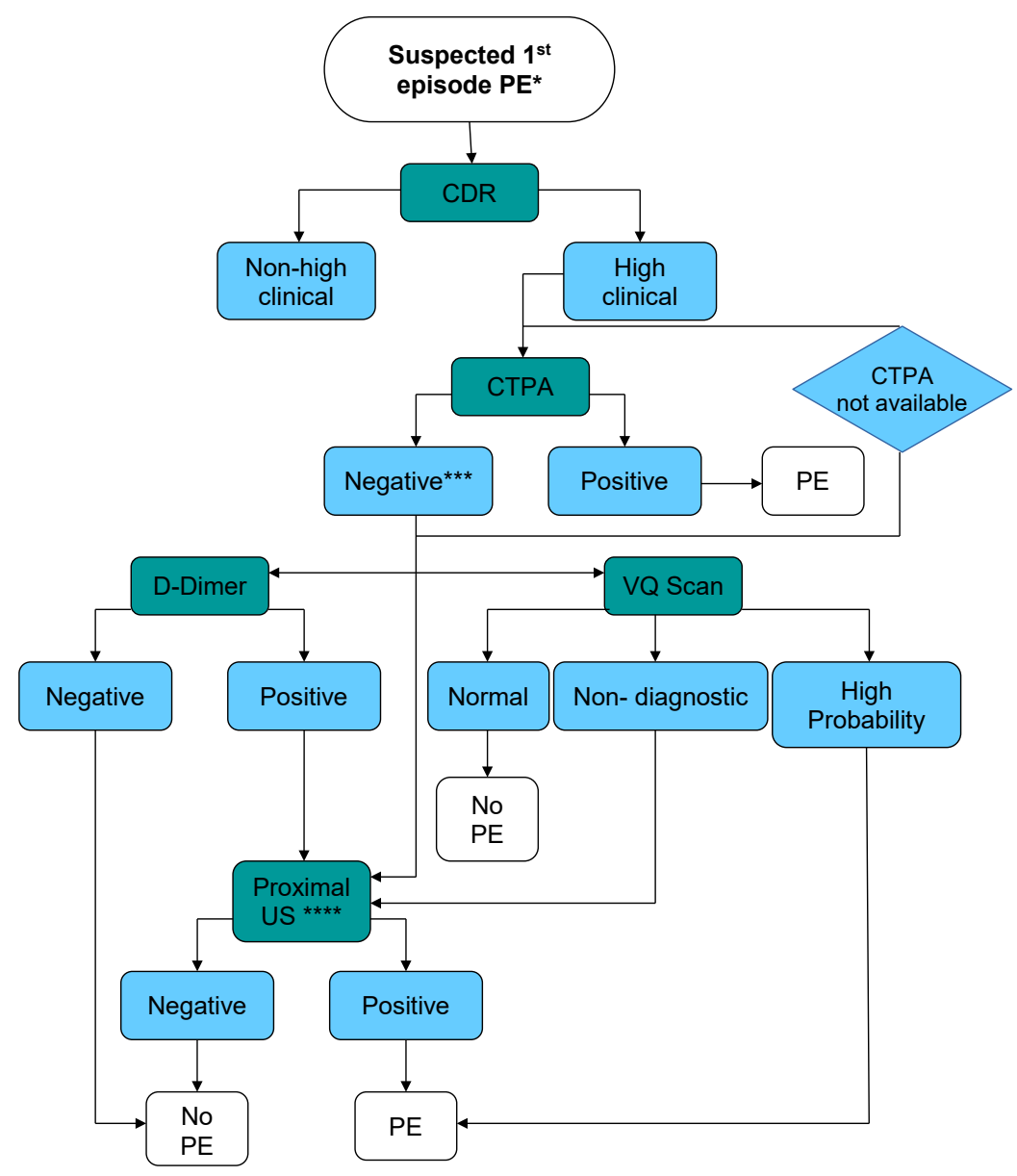

Figure 1: Algorithm for the diagnosis of PE based on a high PTP7

*Hemodynamically stable, nonpregnant patient. ${ }^{*}$ See other algorithms. ***In selected situations, negative CTPA may be enough to rule out PE. ****Serial proximal US if clinical PTP > 50\%.

\section{Management}

The management of PE is based on the prognostic classification and there are many ways of determining severity, some of which requires further special investigations. ${ }^{3}$ The simplified pulmonary embolism severity index (sPESI) is probably the one most useful for the primary care physician. This index attributes one point for each of the following criteria: age $>80$ years, active cancer, chronic cardiopulmonary disease, pulse $\geq 110$ beats per minute, Systolic Blood Pressure $<100 \mathrm{mmHg}$ and oxygen saturation of less than $90 \%$. The patient is considered high risk if the score is one or more. Additional investigations such as Computed Tomography (CT) can improve the prognostic value of the SPESI by assessing right ventricular dysfunction. ${ }^{10}$ However, bedside echocardiography is a much better modality for assessing RV dysfunction (RVD) especially in intermediate and high risk patients and has been found to be as good as CT. ${ }^{11}$ Meinel et al. ${ }^{12}$ demonstrated that a right ventricle to left ventricle (RV/LV) diameter ratio of greater than 0.9 is associated with a 2.5 fold risk of all-cause mortality. In 2009, Bova and colleagues ${ }^{13}$ suggested that the additional measurements of cardiac biomarkers may further aid in risk stratification. Bova and colleagues ${ }^{14}$ developed a predication model for intermediate risk patients using a combination of parameters listed in Table 4.

The Bova model further stages patients into Stage 1 (0-2 points), Stage 2 (3-4 points) and Stage 3 (5-7 points) as shown in Table 5. 
Table 4: Risk Scores based on the Bova Model ${ }^{14}$

\begin{tabular}{lc}
\hline Predictor & Points \\
SBP 90-100 mmHg & 2 \\
Elevated cardiac troponin & 2 \\
RVD (echocardiogram or CT scan) & 2 \\
Heart rate > 110 beats per min & 1 \\
\hline
\end{tabular}

Table 5: The Bova staging system ${ }^{14}$

\begin{tabular}{lccc}
\hline & \multicolumn{3}{c}{ Stage } \\
\cline { 2 - 4 } & I & II & III \\
\hline Points & $0-2$ & $3-4$ & $5-7$ \\
Patients \% & 75.5 & 18.6 & 5.8 \\
$\begin{array}{l}\text { 30-day PE-related } \\
\text { complications \% }\end{array}$ & 4.2 & 10.8 & 29.2 \\
\hline $\begin{array}{l}\text { In-hospital PE-related } \\
\text { complications \% }\end{array}$ & 3.6 & 9.7 & 28.0 \\
\hline 30-day PE-related mortality \% & 1.7 & 5.0 & 15.5 \\
\hline
\end{tabular}

The staging system further prognosticates the 30 day PE-related complications ( $29.2 \%$ in Stage 3), in hospital mortality due to PE (28\% in Stage 3) and the 30 day mortality (15.5\% in Stage 3). ${ }^{14}$ This model was validated and found to have good predictive performance [area under the Receiver Operating Characteristic of 0.73$](\mathrm{Cl} 0.68-0.77) .^{14}$

\section{Therapeutic options}

Patients with hypotension or shock should be stabilised by providing haemodynamic and ventilatory support. Such patients are immediately classified as high risk and are eligible for reperfusion therapy. Stabilisation may require the use of inotropic agents and oxygen delivery devices which may include mechanical ventilation. If mechanical ventilation is contemplated, use low tidal volumes $(6 \mathrm{ml} / \mathrm{kg})$ and aim to keep the end inspiratory plateau pressure $<30 \mathrm{cmH}_{2} \mathrm{O}$. Unfractionated heparin (UFH) is recommended when primary reperfusion is contemplated and in patients with severe renal impairment and those with obesity. Use low molecular weight heparin (LMWH) in low and intermediate-risk patients. Start oral anticoagulants at the same time as the heparin and if using warfarin aim for an International Normalized Ratio (INR) of between two to three. ${ }^{5}$ The novel oral anticoagulants (NOACs) have been approved for use for VTE in Europe and have been found to be equally effective to warfarin with few bleeding incidents but are far more expensive in the South African environment. ${ }^{5,15}$

Thrombolytic treatment is recommended in patients with hypotension and shock. One needs to ensure that judicious screening of patients occurs to exclude contraindications. When using streptokinase, UFH needs to be stopped. Most patients respond well to thrombolysis, but careful monitoring for bleeding is needed. Surgical embolectomy is an alternative modality of treatment when thrombolysis is contraindicated

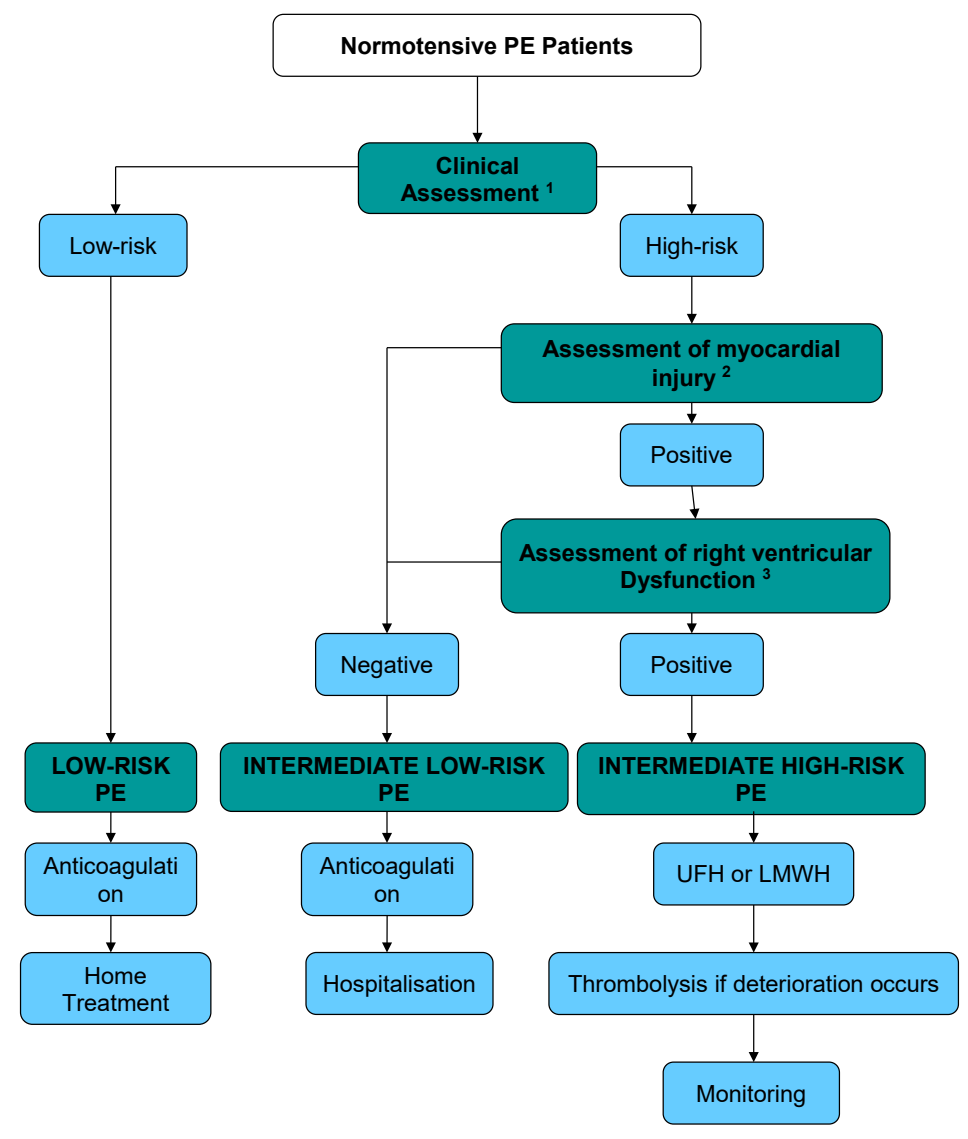

Figure 2: Stepwise algorithm for managing a normotensive patient with $\mathrm{PE}^{3}$

$1=$ using SPESI, $2=$ measure cardiac troponins, $3=$ use echocardiography * patients with concomitant DVT 
or has failed. Per-cutaneous catheter directed embolectomy is an alternative to surgical embolectomy. The surgical and per-cutaneous options may be limited in resource constrained environments. The 2014 ESC guideline does not recommend initial thrombolysis in haemodynamically stable patients, but close monitoring in the intermediate-high risk group is needed to allow for early detection of haemodynamic compromise and appropriate intervention. ${ }^{5}$ In patients without shock or hypotension, treatment involves the initiation of $\mathrm{LMWH}$ and oral anticoagulants. One may choose to use the NOACs if the patient does not have renal impairment. ${ }^{5}$ The Bova prediction model may allow one to discharge a low risk patient early and monitor the patient as an outpatient. ${ }^{14}$ Patients with recurrent PE should be considered for infra-renal venous filters. ${ }^{5}$ Figure 2 developed by Barios ${ }^{3}$ provides a clinical flowchart for managing an intermediate or low risk patient with PE.

For patients with transient risk factors for VTE, anticoagulant therapy should be discontinued after three months. Anticoagulation of indefinite duration is recommended in patients with a second episode of unprovoked PE. Patients on long term anticoagulants need to be assessed regularly to justify the risk-benefit ratio. In patients with cancer and $\mathrm{PE}$, one should consider using LMWH for 3-6 months and anticoagulants for an indefinite period or until the cancer is cured. ${ }^{5}$

\section{Conclusion}

Accurate data of PE contribution to morbidity and mortality in South Africa is not known but a primary care physician needs to consider this diagnosis in a patient presenting with acute cardio-respiratory compromise. Using clinical probability scores will allow one to asses risk and follow a diagnostic algorithm. Once diagnosis is confirmed, prognostic markers establishing risk will determine therapeutic options. The use of D-dimer tests and bedside compression ultrasound and echocardiography is increasingly being used in emergency centres in South Africa and primary care physicians are encouraged to acquire these ultrasound skills which may be invaluable in the emergency assessment of the severely ill patient. Using therapeutic algorithms will allow a decision flow tree which will reduce the length of hospital stay.

\section{References}

1. Torbicki A, Perrier A, Konstantinides S, Agnelli G, Galiè N, Pruszczyk P, et al. Guidelines on the diagnosis and management of acute pulmonary embolism: the Task Force for the Diagnosis and Management of Acute Pulmonary Embolism of the European Society of Cardiology (ESC). Eur Heart J. 2008;29(18):2276-315.

2. Tapson VF. Acute pulmonary embolism. N Engl J Med. 2008;358(10):1037-52.

3. Barrios $D$, Yusen $R D$, Jiménez $D$, editors. Risk stratification for proven acute pulmonary embolism: what information is needed? Seminars in respiratory and critical care medicine. Thieme Medical Publishers; 2017.

4. Awolesi $D$, Naidoo $M$, Cassimjee MH. The profile and frequency of known risk factors or comorbidities for deep vein thrombosis in an urban district hospital in KwaZulu-Natal. South Afr J HIV Med. 2016;17(1):425.

5. Konstantinides SV, Torbicki A, Agnelli G, Danchin N, Fitzmaurice D, Galiè N, et al. 2014 ESC Guidelines on the diagnosis and management of acute pulmonary embolism: The Task Force for the Diagnosis and Management of Acute Pulmonary Embolism of the European Society of Cardiology (ESC) Endorsed by the European Respiratory Society (ERS). 2014;35(43):3033-73.

6. Guo DJ, Zhao C, Zou YD, Huang XH, Hu JM, Guo L. Values of the Wells and revised Geneva scores combined with $D$-dimer in diagnosing elderly pulmonary embolism patients. Chin Med J (Engl). 2015;128(8):1052-7.

7. Lim W, Le Gal G, Bates SM, Righini M, Haramati LB, Lang E, et al. American Society of Hematology 2018 guidelines for management of venous thromboembolism: diagnosis of venous thromboembolism. Blood Adv. 2018;2(22):3226-56.

8. Filopei J, Acquah SO, Bondarsky EE, Steiger DJ, Ramesh N, Ehrlich M, et al. Diagnostic Accuracy of Point-of-Care Ultrasound Performed by Pulmonary Critical Care Physicians for Right Ventricle Assessment in Patients With Acute Pulmonary Embolism. Crit Care Med. 2017;45(12):2040-5.

9. Laursen CB, Sloth E, Lambrechtsen J, Lassen AT, Madsen PH, Henriksen DP, et al. Focused sonography of the heart, lungs, and deep veins identifies missed life-threatening conditions in admitted patients with acute respiratory symptoms. Chest. 2013;144(6):1868-75.

10. Jimenez D, Lobo JL, Monreal M, Moores L, Oribe M, Barron M, et al. Prognostic significance of multidetector $C T$ in normotensive patients with pulmonary embolism: results of the protect study. Thorax. 2014;69(2):109-15.

11. Sanchez O, Trinquart L, Colombet I, Durieux P, Huisman MV, Chatellier G, et al. Prognostic value of right ventricular dysfunction in patients with haemodynamically stable pulmonary embolism: a systematic review. Eur Heart J. 2008;29(12):1569-77.

12. Meinel FG, Nance JW, Jr., Schoepf UJ, Hoffmann VS, Thierfelder KM, Costello P, et al. Predictive Value of Computed Tomography in Acute Pulmonary Embolism: Systematic Review and Meta-analysis. Am J Med. 2015;128(7):747-59 e2.

13. Bova C, Pesavento R, Marchiori A, Palla A, Enea I, Pengo V, et al. Risk stratification and outcomes in hemodynamically stable patients with acute pulmonary embolism: a prospective, multicentre, cohort study with three months of follow-up. JThromb Haemost. 2009;7(6):938-44.

14. Bova C, Sanchez O, Prandoni P, Lankeit M, Konstantinides S, Vanni S, et al. Identification of intermediate-risk patients with acute symptomatic pulmonary embolism. Eur Respir J. 2014;44(3):694-703.

15. Laas DJ, Naidoo M. Oral anticoagulants and atrial fibrillation: A South African perspective. S Afr Med J. 2018;108(8):640-6. 